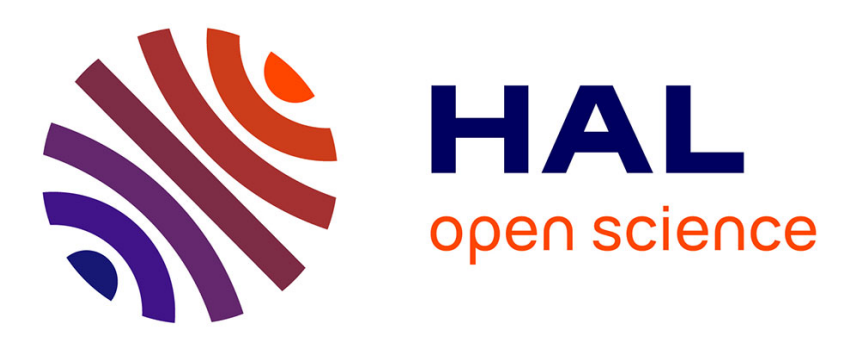

\title{
Mise en évidence de la néogenèse de sables calcaires sous l'influence des racines : incidence sur la granulométrie du sol
}

Benoît Jaillard

\section{To cite this version:}

Benoît Jaillard. Mise en évidence de la néogenèse de sables calcaires sous l'influence des racines: incidence sur la granulométrie du sol. Agronomie, 1984, 4 (1), pp.91-100. hal-00884615

\section{HAL Id: hal-00884615 https://hal.science/hal-00884615}

Submitted on 1 Jan 1984

HAL is a multi-disciplinary open access archive for the deposit and dissemination of scientific research documents, whether they are published or not. The documents may come from teaching and research institutions in France or abroad, or from public or private research centers.
L'archive ouverte pluridisciplinaire HAL, est destinée au dépôt et à la diffusion de documents scientifiques de niveau recherche, publiés ou non, émanant des établissements d'enseignement et de recherche français ou étrangers, des laboratoires publics ou privés. 


\title{
Mise en évidence de la néogenèse de sables calcaires sous l'influence des racines: incidence sur la granulométrie du
} sol

Benoît JAILLARD

I.N.R.A., Laboratoire de Science du Sol, E.N.S.A. 9, place Viala, F 34060 Montpellier Cedex

RÉSUMÉ

\begin{abstract}
L'étude microstructurale d'un sol calcaire issu de l'altération de marnes révèle la présence de nombreuses micro-structures calcaires se développant au niveau des discontinuités fissurales. Il s'agit de vestiges de racines de graminées, constitués de cellules calcifiées d'environ $100 \mu \mathrm{m}$ de diamètre, indépendantes les unes des autres mais ayant conservé leur arrangement tissulaire.

D'un autre côté, l'analyse granulométrique détaillée des sables fins $(50-200 \mu \mathrm{m})$ met en évidence un pic aux alentours de $80 \mu \mathrm{m}$. L'observation des fractions granulométriques correspondantes, à la loupe binoculaire et au microscope à balayage électronique, nous permet de distinguer (par leur forme et leur couleur) les sables cytomorphes des sables hérités de la marne et de quantifier (par comptage) les proportions de chacune des deux formes.

Il apparaît ainsi que la calcification des cellules des racines est à l'origine de sables (cytomorphes) qui constituent jusqu'au quart de la phase minérale du sol au voisinage de la surface.
\end{abstract}

Mots clés additionnels : Pédologie structurale, climat méditerranéen, sol brun calcaire, calcite, chlorose.

SUMMARY

Evidence for the neogenesis of calcareous sands under the influence of roots : incidence on soil granulometry.

A microstructural study of calcareous soil from marl weathering showed the presence of calcarcous features developing along structural discontinuities. These corresponded with the traces of roots, composed of calcified cells of size ca $100 \mu \mathrm{m}$, still arranged in a tissue, but independent one from another. The tissues concerned were mostly the epidermis, the cortical parenchyma and the endodermis. The central cylinder was generally represented by a gap.

Detailed granulometric analysis of the fine sand fraction $(50-200 \mu \mathrm{m})$ showed a peak at about $80 \mu \mathrm{m}$. The observation of granulometric fractions both with binocular lens and by scanning electron microscopy allowed the distinction (by shape and colour) of cytomorphic sands from sands coming from the parent material and their quantification (by counting). The cytomorphic sands were sparitic calcite crystals.

The calcification of root cells thus apparently gives rise to cytomorphic sands forming up to quarter of the mineral phase of the soil near the surface, a silt-sandy soil developing from a silt-clayey marl.

Additional key words : Structural pedology, mediterranean climate, cambisol, calcite, chlorosis.

\section{INTRODUCTION}

Tout en assurant leur fonction première de nutrition minérale et d'alimentation en eau de la plante, les racines ont une action sur le sol qui revêt des aspects très variés selon les milieux et les conditions physico-chimiques régnant dans ces milieux. Cette action a pour effet principal une réorganisation des constituants du sol avec des conséquences d'ordre pédologique, physiologique et agronomique. Dans un récent ouvrage, CALLOT et al. (1983) envisagent les diverses facettes de ces « interactions sol-racine".
Ces auteurs soulignent l'importance de l'analyse structurale de la rhizosphère, car la réorganisation du sol par les racines peut considérablement modifier l'environnement physicochimique de la racine.

En milieu carbonaté, l'action structurale des racines est généralement très marquée du fait de la relative solubilité des carbonates. L'absorption racinaire engendrant des flux hydriques convergeant vers la racine, cette activité se manifeste le plus souvent par une accumulation périracinaire de carbonates qui, selon les conditions du milieu, se présentent sous forme d'aiguilles de calcite, de calcaire fin 
dit " micritique " ou de concrétions calcaires dures tubulaires entourant la racine (WIEDER \& YAALON, 1982 ; CALLOT et al., 1983).

Mais la racine elle-même est le siège de nombreux processus de concentration minérale : cristaux d'oxalate de calcium dans les vacuoles, potassium et calcium dans les parois cellulaires (CHINO, 1981; GHORBAL, 1979). Certains de ces processius sont susceptibles de contribuer à la transformation des constituants mêmes du sol. Ainsi différents auteurs (MEYER, 1981; BUTEL, 1982; JAILLARD, 1983) ont observé la calcification des cellules du cortex de racines.

Dans cette note, nous allons montrer que ce phénomène étonnant doit être considéré sous un angle pédogénétique, car susceptible de modifier notablement la granulométrie du sol.

\section{PRÉSENTATION DU SITE}

La présente étude a été réalisée sur le domaine expérimental de Coulondres, au lieu-dit Bois des Grasses, commune de Saint-Gély-du-Fesc, Hérault. Le climat est de type méditerranéen $(\mathrm{P}=1100 \mathrm{~mm} ; \mathrm{ETP}=1100 \mathrm{~mm})$ à fort contraste saisonnier. La végétation est une garrigue dite «à romarin », association végétale buissonnante très clairsemée, où dominent le romarin (Rosmarinus officinalis L.), le chêne kermès (Quercus ilex L.) et le genévrier oxycèdre (Juniperus oxycedrus L.). On y rencontre également par place le pin d'Alep (Pinus halepensis Mill.). Cet écosystème, très pauvre, est traditionnellement utilisé comme «parcours» pour les troupeaux ovins.

Les garrigues «à romarin " sont caractéristiques des zones marno-calcaires. Le domaine de Coulondres repose sur lc Valanginien (Crétacé inférieur), formation sédimentaire marine constituée de marnes calcaires jaunes à grises en alternance avec des bancs calcaires plus ou moins marneux d'épaisseur centimétrique à décamétrique. Cette alternance de bancs plus ou moins durs engendre un relief peu accusé de cuestas et ensellements (fig. 1a).

Une carte des sols au $1 / 2500^{\mathrm{e}}$ a été établie par MEYER (1981), à la suite d'une analyse géo-pédologique de la région. L'évolution des sols est directement liée à la dureté du substratum calcaire. Sur les bancs calcaires durs des cuestas, les sols sont décarbonatés, argileux et bien structurés (sols bruns calciques). En revanche, les sols développés

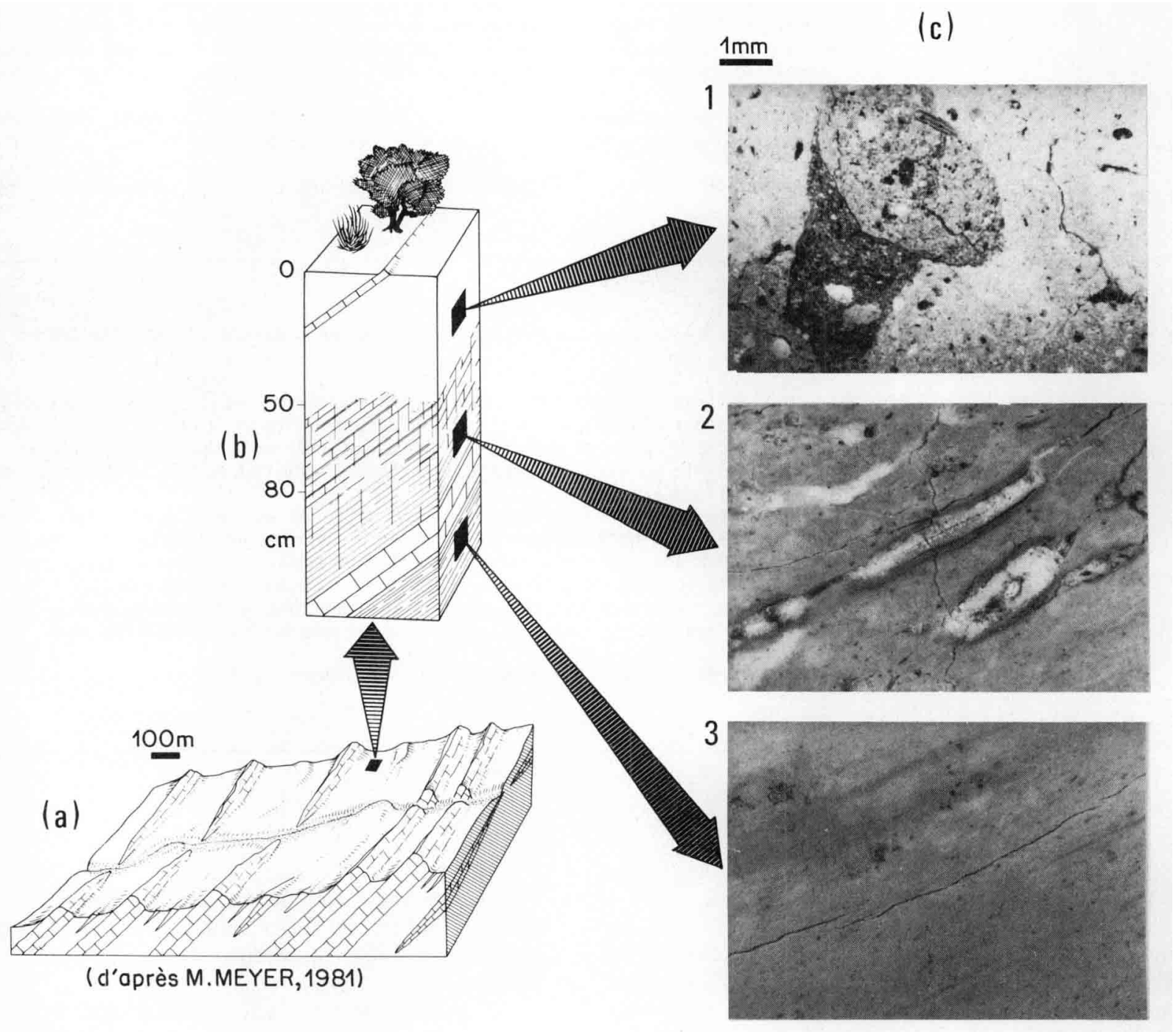

Figure 1

Morphologie struciurale du sol.

(a) Structure sédimentaire régionale: marnes calcaires et bancs calcaires marneux en alternance.

(b) Profil de sol développé sur marnes calcaires.

(c) Micromorphologie des horizons du sol.
Structural morphology of the soil.

(a) Regional sedimentary structure: calcareous marls and marly calcareous banks alternating.

(b) Profile of soil developed from calcareous marls.

(c) Micromorphology of soil horizons. 
sur les marnes calcaires n'ont pas subi de décarbonatation significative : leurs teneurs en $\mathrm{CaCO}_{3}$ oscillent, comme dans le matériau originel, entre 0,80 et $0,90 \mathrm{~g} / \mathrm{g}$. Nous allons nous intéresser à ces sols très calcaires (sols bruns calcaires).

Le site choisi se trouve au creux d'un ensellement d'une centaine de mètres de large (fig. 1b). Les marnes calcaires y alternent avec des bancs isolés, centimétriques à décimétriques de calcaire marneux tendre et fortement diaclasé, espacés de quelques mètres. Le pendage est de l'ordre de $25^{\circ}$. Ce site présente les unités de sols les plus défavorables à la végétation.

Le sol peut être décomposé en 3 horizons caractérisés par le degré d'altération du matériau originel:

- en profondeur ( $80 \mathrm{~cm}$ et au-delà), les marnes sont peu fissurées, massives et compactes $\left(\rho d=2,0 \mathrm{~g} / \mathrm{cm}^{3}\right)$, à cassure conchoïdale (fig. 1c, photo 3 ) ;

— de 80 à $50 \mathrm{~cm}$, ces marnes, plus fissurées $(\rho \mathrm{d} \simeq$ $1,80 \mathrm{~g} / \mathrm{cm}^{3}$ ), se débitent selon les plans de sédimentation (fig. 1c, photo 2) ;

- de $50 \mathrm{~cm}$ à la surface du sol, la structure litée disparaît pour devenir continue $\left(\rho \mathrm{d} \simeq 1,65 \mathrm{~g} / \mathrm{cm}^{3}\right)$, le matériau ayant été brassé par l'activité des lombriciens (fig. 1c, photo 1).

Le sol apparaît donc, au premier abord, comme résultant pour l'essentiel de la désorganisation de la structure litée des marnes sédimentaires, sans autre altération, pas même décarbonatation du matériau. Le profil reste compact, massif, mal aéré et fort peu perméable.

En fait, le trait morphologique dominant est la présence générale et en grand nombre de «pseudo-mycéliums calcaires »: ce sont de fins rubans blancs d'1 à $2 \mathrm{~mm}$ de large, isolés, enchevêtrés ou ramifiés. Un examen à la loupe révèle qu'il s'agit en réalité de racines calcifiées. L'abondance dans le profil de ces structures, observées et décrites par MEYER (1981), a été utilisé par cet autcur comme l'un des critères de différenciation cartographique des sols. Le site étudié dans cette note a été choisi en conséquence, au sein d'une unité dans laquelle le phénomène est important.

$\mathrm{Au}$ terme de pseudo-mycéliums calcaires, nous préférerons celui plus explicite de «structures rhizomorphes».

\section{ANALYSE DES RÉSULTATS}

\section{A. Etude morphologique}

\section{Localisation des structures rhizomorphes}

Les structures rhizomorphes sont d'une manière très générale localisées dans les plans de fracturation du matériau : fissures, lits de sédimentation ou microfissures interagrégats (fig. 1c, photo 2) :

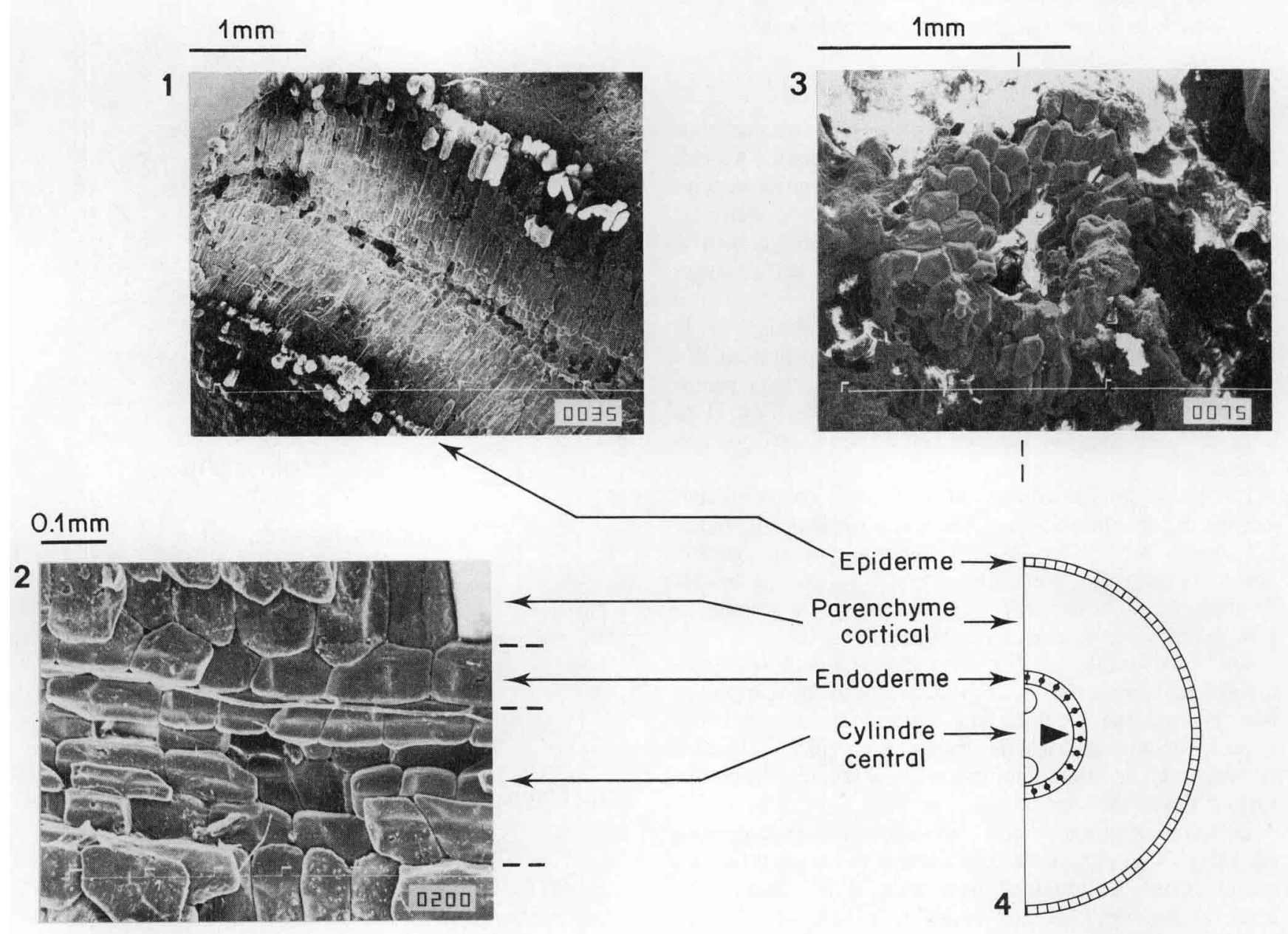

Figure 2

Morphologie des structures rhizomorphes.

(1) Coupe longitudinale.

(2) Détail d'une coupe longitudinale.

(3) Coupe transversale.

(4) Représentation schématique de l'anatomie d'une racine, en coupe transversale.
Morphology of the rhizomorphic structures.

(1) Longitudinal section.

(2) Detail of a longitudinal section.

(3) Tranverse section.

(4) Schematic representation of root anatomy, in transverse section. 
- En profondeur $(110-80 \mathrm{~cm})$, les microstructures, assez rares, se développent exclusivement dans les plans de fissures verticales et les premiers centimètres adjacents des plans de sédimentation, c'est-à-dire dans les zones où le matériau commence à s'altérer. Les quelques racines vivantes exploitent les mêmes microsites fissuraux.

- De 80 à $50 \mathrm{~cm}$, les plans de sédimentation s'ouvrent progressivement tandis que les fissures verticales deviennent plus nombreuses : les structures rhizomorphes, de plus en plus abondantes, très ramifiées et souvent enchevêtrées, sont localisées dans ces discontinuités porales. Cet horizon est d'ailleurs le plus caractéristique du fait du débit du matériau selon les plans de sédimentation et de la forte densité de racines calcifiées à leur niveau.

- De 50 à $30 \mathrm{~cm}$, les structures rhizomorphes sont moins abondantes et très tortueuses : elles suivent les facettes des agrégats ou les parois de trous de lombriciens colmatés.

- Enfin leur nombre chute rapidement dans les 20 premiers centimètres, pour devenir négligeable au voisinage immédiat de la surface du sol.

Ainsi, les structures calcaires épousent exactement l'organisation fissurale du matériau : microfissures, facettes interagrégats, parois de trous colmatés, toutes discontinuités structurales susceptibles de favoriser la pénćtration actuelle des racines. La distribution dans le profil des structures rhizomorphes et des racines vivantes sont strictement identiques. Ce fait suggère fortement que la genèse des structures rhizomorphes est un processus actuel.

\section{Morphologie des structures rhizomorphes}

Ces structures rhizomorphes se présentent, comme nous l'avons signalé, sous forme de fins rubans blancs d' 1 à $2 \mathrm{~mm}$ de large environ. Leur observation à faible grossissement révèle leur anatomie cellulaire : chaque vestige racinaire est constitué par l'imbrication de cellules calcifićes d'environ $100 \mu \mathrm{m}$, indépendantes, mais ayant conservé leur arrangement tissulaire.

Les tissus les mieux calcifiés sont l'épiderme et le parenchyme cortical. L'endoderme est souvent présent. En revanche les tissus médullaires : xylème, phloème et parenchyme médullaire ne sont que rarement minéralisés. Dans la plupart des cas, le cylindre central est représenté par une lacune.

Les photographies composant la figure 2 correspondent aux racines calcifiées les plus fréquentes sur le profil étudié. L'anatomie de ces racines se caractérise par un extrême développement du parenchyme cortical qui occupe les $3 / 4$ du diamètre de la racine. Il s'agit de racines de graminées, probablement représentées par plusieurs espèces.

- Chaque cellule calcifiée étant indépendante, les structures rhizomorphes se désorganisent aisément. L'observation micromorphologique, sous binoculaire et sur lames minces de sol, permet de mettre en évidence l'action perturbatrice des eaux d'infiltration, des racines vivantes et surtout des lombriciens.

La lacune centrale de ces micro-structures, pore continu de 200 à $500 \mu \mathrm{m}$ de diamètre, s'avère souvent constituer un cheminement préférenticl des eaux d'infiltration. On observe dans ce cas des zones d'érosion des cellules calcifiées. Les racines vivantes, lors de leur croissance, affectent également ces microstructures. Dans la plupart des cas, il s'agit d'une désorganisation sans transport notable des cellules. Quant aux lombriciens, nous verrons plus loin le rôle important qu'ils jouent dans ce sol.

- Du point de vue minéralogique, les cellules calcifiées se caractérisent par leur cristallisation sparitique (macro-cris- taux) et leur pureté optique : sous binoculaire, elles prennent un aspect hyalin ou blanc opalescent. Elles sont constituées, pour plus de 99 p. 100 , de calcite. Ces cellules calcifiées présentent une surface lisse, une forme polyédrique arrondie (fig. 3). Le microscope à balayage ćlectronique révèle parfois une altération de surface mettant en évidence leur structure macrocristalline (sparite). Ceci explique la diffraction de la lumière (opalescence) observée à la loupe binoculaire pour certaines cellules.
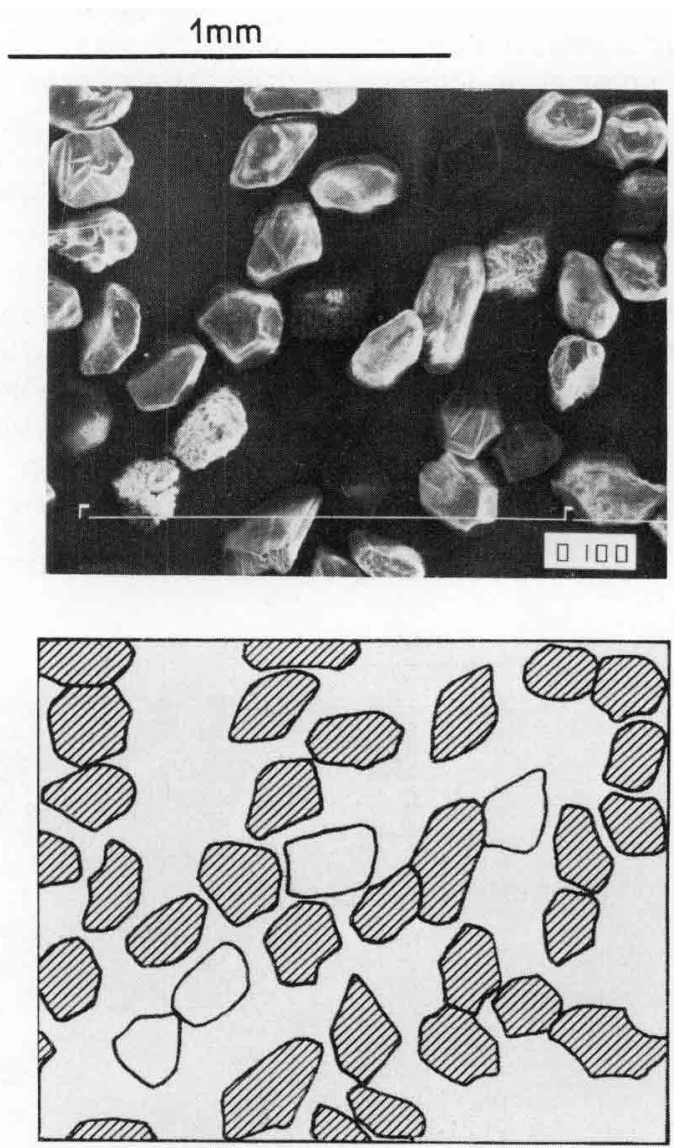

\section{Sables cytomorphes}

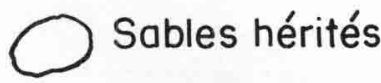

Figure 3

Morphologie des sables : fraction $105-125 \mu m$ à $60-70 \mathrm{~cm}$ de profondeur.

Morphology of sands : $105-125 \mu \mathrm{m}$ size fraction at $60-70 \mathrm{~cm}$ depth.

\section{B. Etude granulométrique}

\section{Méthodes}

MEYER (1981) avait constaté que les sols bruns calcaires sur marnes calcaires étaient plus riches en sables en surface qu'en profondeur. Nous devons nous demander si ces sables ne seraient pas d'origine cellulaire. Il convient donc de quantifier l'importance réelle du phénomène précédemment mis en évidence.

Un matériau marneux se prête particulièrement bien à ce type de recherche. Les marnes sédimentaires marines présentent un faciès granulométrique dit «hyperbolique » 

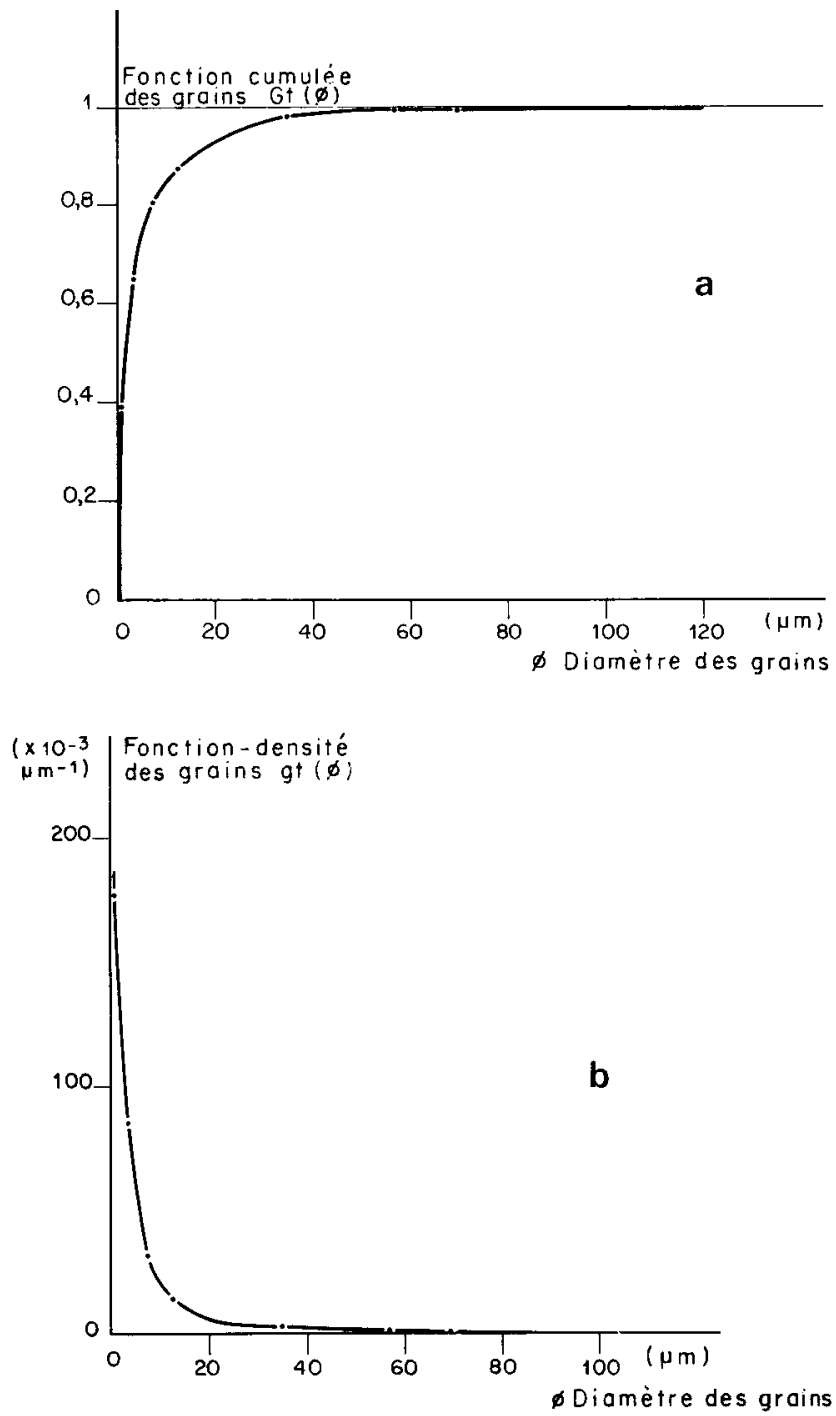

Figure 4

Faciès granulométrique des marnes calcaires.

(a) Courbe cumulée Gt( $\varnothing)$.

(b) Fonction-densité gt $(\varnothing)$, dérivée première de la courbe cumulée $G t(\varnothing)$.

Granulometric facies of calcareous marls.

(a) Cumulative-function $\mathrm{Gt}(\varnothing)$

(b) Density-function gt( $\varnothing)$, first derivative of the cumulative function $G t(\varnothing)$

typique (fig. 4a). Ainsi, le taux massique de grains supérieurs à $20 \mu \mathrm{m}$ n'excède pas 10 p. 100 et surtout le taux de grains supérieurs à $50 \mu \mathrm{m}$ est négligeable, de l'ordre de 3 p. 100 de la masse des sédiments. La néogenèse de sables fins $(50 \mu \mathrm{m} \leqslant \varnothing<200 \mu \mathrm{m})$, même en faible quantité, pcut donc être aisément détectée.

La taille des cellules calcifiées étant d'environ $100 \mu \mathrm{m}$, une séparation granulométrique par tamisage autour de cette valeur nous permet d'analyser l'évolution sur le profil du faciès granulométrique des sables fins. De 50 à $200 \mu \mathrm{m}$, les mailles de tamis disponibles s'échelonnent tous les 10-20 $\mu \mathrm{m}$ : un fractionnement important des sables fins est réalisable ( 7 classes sur $130 \mu \mathrm{m}$ ).

Par ailleurs, les grains origincls d'unc taille supérieure à $50 \mu \mathrm{m}$ sont représentés par quelques quartz et oxydes métalliques mais, pour l'essentiel, par des carbonates sous forme de pelotes ou plaquettes micritiques, de teinte jaunâtre à brun sale. Les sables cytomorphes s'en distinguent nettement, comme nous l'avons vu, par suite de leur pureté optique.

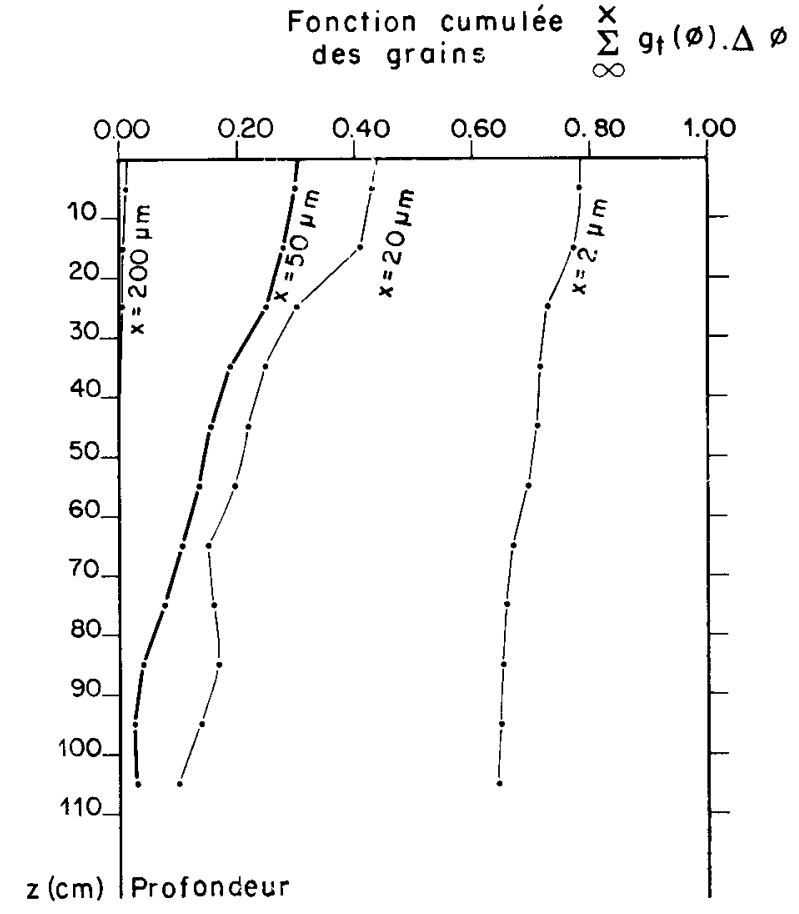

Figure 5

Profil granulométrique global du sol.

Overall granulometric soil profile.

La détermination de la proportion de l'une et l'autre forme, sédimentaire et cytomorphe, a ainsi été réalisée sur chaque fraction granulométrique, par comptage sous binoculaire (semis sur papier millimétré et comptage d'environ 500 grains par fraction). Les résultats granulométriques sont cxprimés en fonction-densité notéc $g(\varnothing)$ (fig. 4b), dérivéc première de la fonction cumuléc notée $G(\varnothing)$ (fig. 4a). Cette expression nous a semblé préférable à d'autres plus couramment cmployées en science du sol, car elle permet de s'affranchir des intervalles irréguliers entre tamis et donc de mettre en évidence des pics ou vallées de distribution des grains.

\section{MODE OPÉRATOIRE \\ ET EXPRESSION DES RÉSULTATS}

- sur environ $50 \mathrm{~g}$ de matériau désagrégé :

- dispersion aux ultra-sons $(10 \mathrm{mn})$ en solution basique $(1 \mathrm{ml}$ de $\mathrm{NH}_{3} / 100 \mathrm{ml}$ ) ;

- élimination des fines par tamisage sous eau à $50 \mu \mathrm{m}$;

- séchage à $110^{\circ} \mathrm{C}$;

- ćlimination de la matic̀re organique par sćdimentation en solution de bromoforme-benzène $\left(\rho \mathrm{d}=2,4 \mathrm{~g} / \mathrm{cm}^{3}\right)$ (massc volumique de la matière organique comprise entre 0,9 ct $2,0 \mathrm{~g} / \mathrm{cm}^{3}$ du fait de sa charge en $\left.\mathrm{CaCO}_{3}\right)$;

- séchage à froid sous hotte ;

- tamisage à sec $(10 \mathrm{mn})$ sur tamis de $180,150,125,105,88,75$, 63 et $50 \mu \mathrm{m}$, soit des classes granulométriques de $18 \mu \mathrm{m}$ d'intervalle en moyenne;

- cxpression des résultats granulométriques en fonction-densité de grains gt $(\varnothing)$ telle que : $\operatorname{gt}(\varnothing)=\mathrm{m}(\varnothing) / \Delta \varnothing$

où : $m(\varnothing)=$ masse de la classe granulométrique $\Delta \varnothing=$ intervalle de la classe granulométrique

avec

$$
\mathrm{Gt}(\varnothing)=\sum_{x=0}^{\varnothing} \operatorname{gt}(\mathrm{x}) \cdot \Delta \mathrm{x}
$$

- les proportions $\alpha(\varnothing)$ sont directement converties en fonctiondensité. Soit :

$$
\operatorname{gt}(\varnothing)=\operatorname{gc}(\varnothing)+\operatorname{gh}(\varnothing)
$$

avec : $\operatorname{gh}(\varnothing)=$ fonction densité partielle de grains cytomorphes $\operatorname{gc}(\varnothing)=$ fonction-densité particlle de grains hérités. 

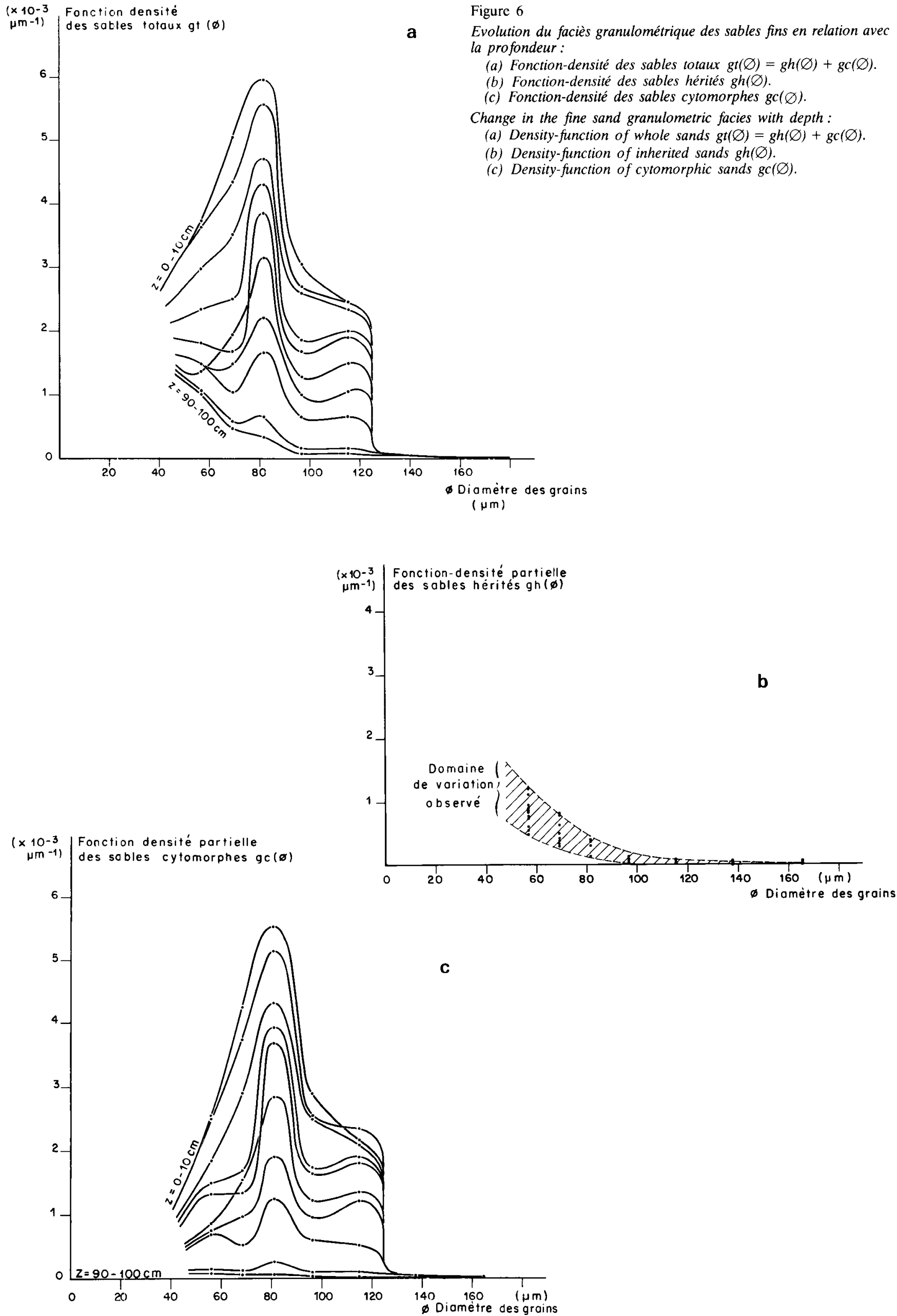
Nous avons :

$$
\begin{aligned}
& \operatorname{gc}(\varnothing)=\alpha(\varnothing) \cdot \operatorname{gt}(\varnothing) \\
& \operatorname{gh}(\varnothing)=[1-\alpha(\varnothing)] \cdot \operatorname{gt}(\varnothing)
\end{aligned}
$$

et :

$$
\mathrm{Gi}(\varnothing)=\sum_{\mathrm{x}=0}^{\varnothing} \mathrm{gi}(\mathrm{x}) \cdot \Delta \mathrm{x} \text { pour } \mathrm{i}=\mathrm{t}, \mathrm{h} \text { ou } \mathrm{c} .
$$

\section{Evolution de la granulométrie du sol}

a) Sables totaux

Globalement, l'évolution granulométrique du sol se caractérise par l'augmentation régulière de la base vers la surface du sol de la fraction sableuse fine (fig. 5), passant de 3 p. 100 à $80 \mathrm{~cm}$ et au-delà, à 30 p. 100 en surface. Il s'agit donc probablement d'un phénomène pédogénétique autochtone et non pas d'une hétérogénéité sédimentaire quelconque ou d'un apport colluvial.

L'étude détaillée de la fraction sableuse précise ce processus (fig. 6a et 7a). Au-delà de $90 \mathrm{~cm}$ de profondeur, le faciès granulométrique des sables n'est autre que celui du matériau originel : il correspond à la queue de distribution hyperbolique du sédiment marno-calcaire. Puis, de $90 \mathrm{~cm}$ à la surface du sol, le faciès se modifie, mettant en évidence la genèse de sables remarquablement calibrés : de 90 à $30 \mathrm{~cm}$ de profondeur, aucun sable n'excède la taille de $125 \mu \mathrm{m}$. En surface, les particules d'une taille supérieure à $125 \mu \mathrm{I}_{\text {. }}$. représentent toujours moins de 1,5 p. 100 de la masse de solide. Le pic de distribution est done strictement borné à $125 \mu \mathrm{m}$.

En deçà de $75 \mu \mathrm{m}$, l'évolution du faciès granulométrique est un peu plus complexe, le pic de distribution ne présentant pas de borne inféricure. Une telle distribution évoque un processus de genèse de sables calibrés vers $75-125 \mu \mathrm{m}$, évoluant secondairement par dissolution.

Ainsi, la composition granulométrique du matériau originel est profondément modifiée par l'apparition de sables fins totaux représentant en surface jusqu'à 30 p. 100 de la masse solide du sol.

\section{b) Sables hérités et sables cytomorphes}

La fonction-densité particlle de sables « hérités » $\operatorname{gh}(\varnothing)$ reste sensiblement constante sur l'ensemble du profil (fig. 6b). En revanche, la fonction-densité partielle de sables cytomorphes $\mathrm{gc}(\varnothing)$ croît continuellement de la base du profil à la surface (fig. $6 \mathrm{c}$ et $7 \mathrm{~b}$ ). Il y a bien, in situ, genèse de sables cytomorphes.
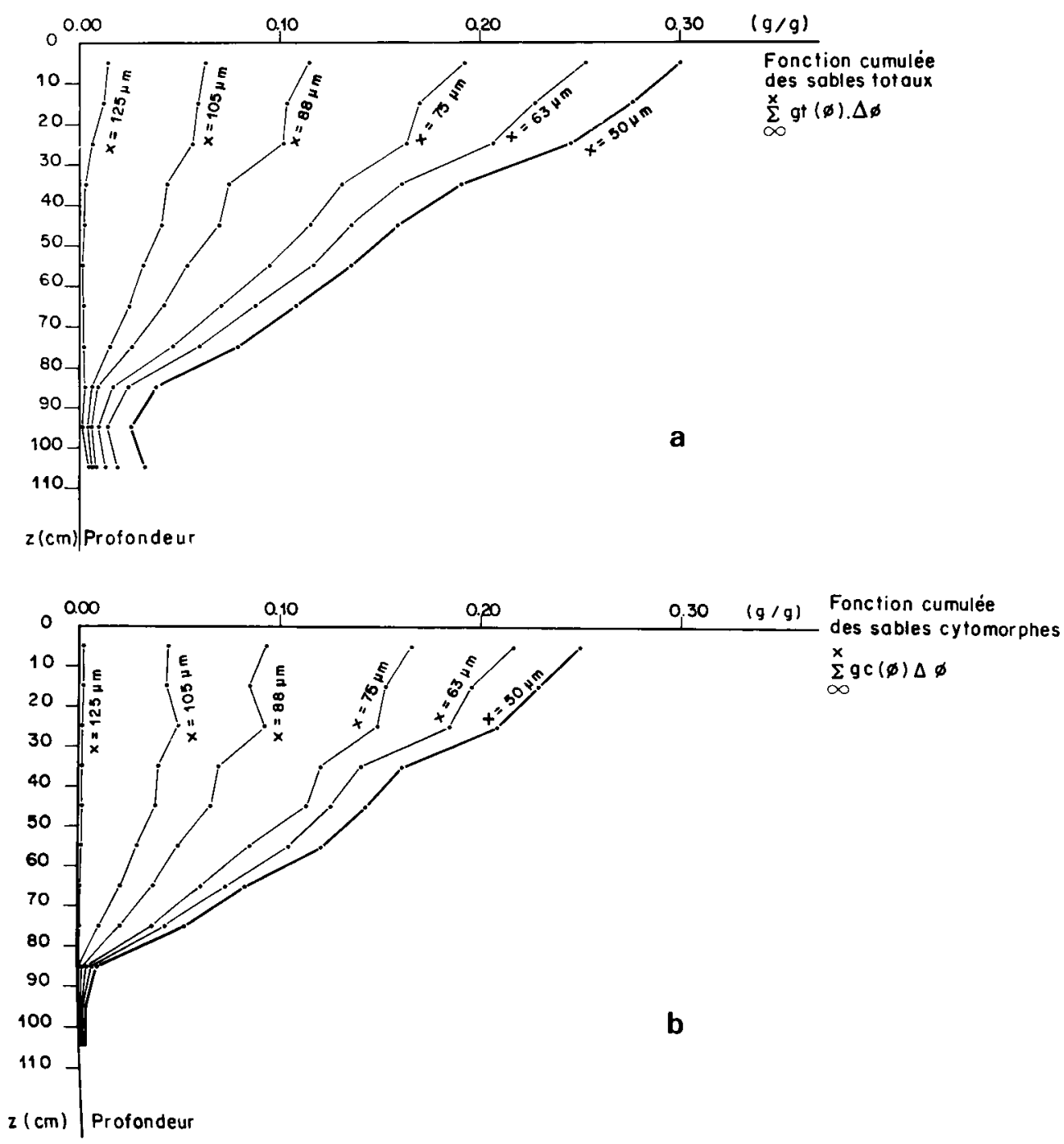

Figure 7

Profil granulométrique des sables fins:

(a) Sables totaux.

(b) Sables cytomorphes. 
Le pic de production est strictement borné à $125 \mu \mathrm{m}$, taille des plus grosses cellules du parenchyme cortical des racines de ces graminćcs. Il présente un maximum vers $80 \mu \mathrm{m}$. Enfin on observe une queue de distribution dans les limons grossicrs $(20 \leqslant \varnothing<50 \mu \mathrm{m})$, d'autant plus importante que l'on s'approche de la surface.

En définitive, il apparait que les sables cytomorphes, de taille supéricure à $50 \mu \mathrm{m}$, représentent en surface 25 p. 100 de la phase solide : il s'agit donc d'un processus pédogénétique essenticl.

\section{Dynamique structurale du sol}

La figure 8 illustre la dynamique de formation du sol. Les proportions de sables organisés en structures rhizomorphes ct de sables incorporés à la matrice du sol ont été estimécs sur des surfaces polies de sol imprégné de résine. Les dessins ont également ćté réalisés à partir de surfaces polies de sol.

\section{a) Action des racines}

L'ensemble des résultats précédents montre qu'au cours du temps les racines participent activement à l'altération du substratum marno-calcaire du Valanginien de Saint-Gélydu-Fesc (fig. 8-1 et fig. 1c, photo 3) et à la formation du sol. En fait, l'action des racines consiste en une redistribution des carbonates à l'échelle du rhizocylindre.

En profondeur, l'observation micro-morphologique révèle que les racines progressent quasi uniquement par

\section{SABLES CYTOMORPHES}

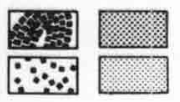

organisés en structures rhizomorphes

désorganisés et incorporés à la matrice du sol
(4)

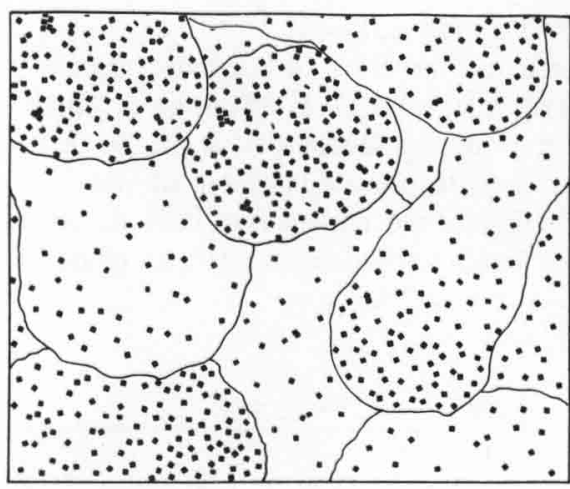

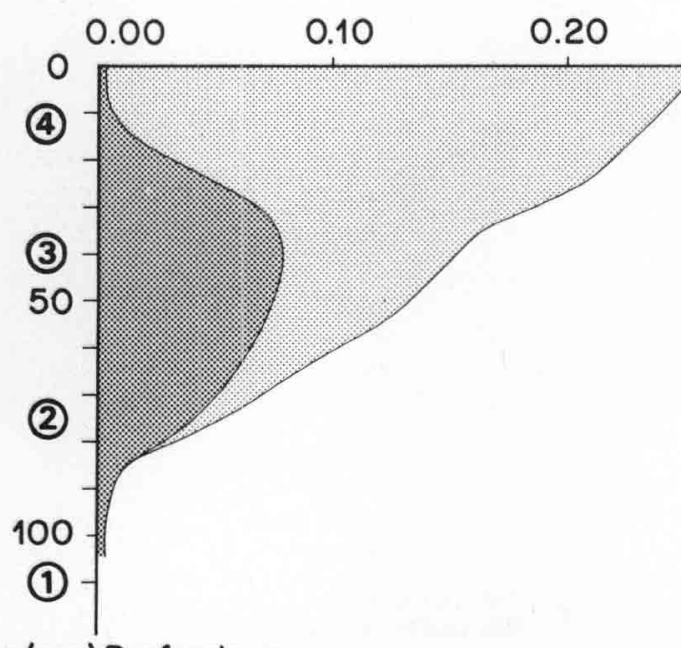

$z(\mathrm{~cm})$ Profondeur
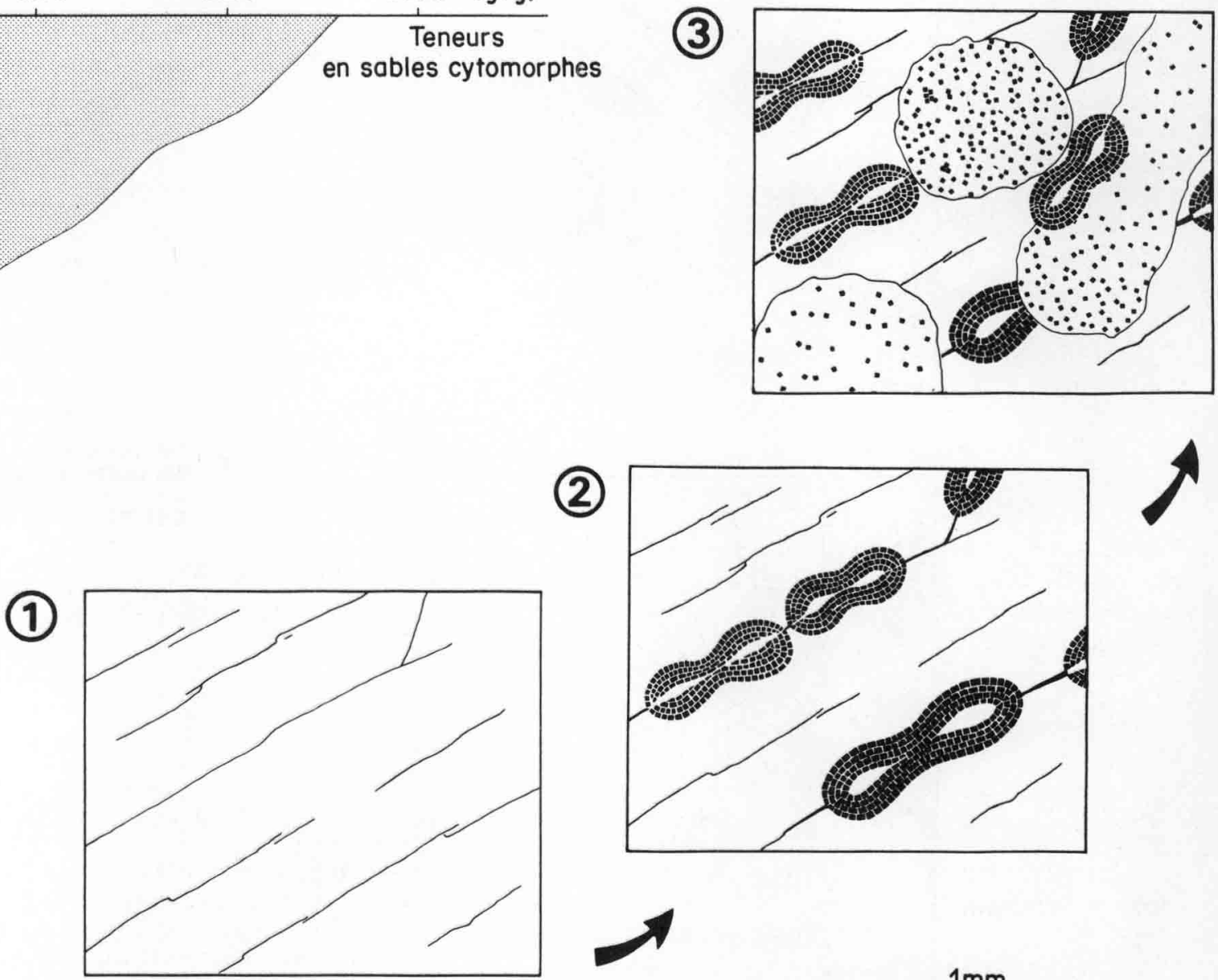

(2)
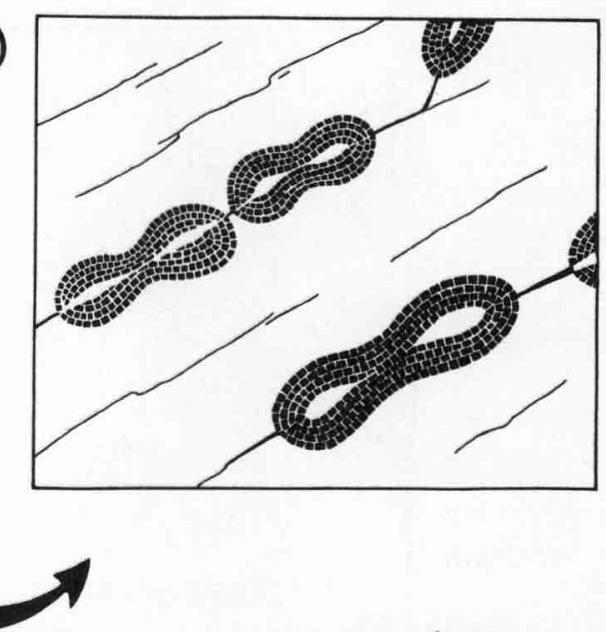

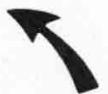
en sables cytomorphes

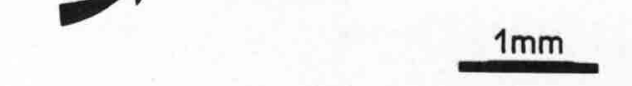

Figure 8

Représentation schématique de l'évolution structurale du sol: effets respectifs de la caicification des racines et du brassage du matériau marneux par les lombriciens.
Schematic representation of the structural evolution of the soil: combined effects of root calcification and of mixing by earth-worms. 
dissolution des carbonates qui occupent de 80 à 90 p. 100 du volume. En effet, les quelques dalles et plaquettes de calcaire marneux dur gardent l'empreinte en creux du passage des racines, le plus souvent encore remplie par leurs vestiges calcifiés. Dans les marnes, la structure sédimentaire n'est guère perturbée par la présence d'une racine : seule une couronne d'environ $200 \mu \mathrm{m}$ d'épaisscur a été fortement décarbonatée par la racine.

Du point de vue structural, il y a donc, au niveau du rhizocylindre, ségrégation des carbonates et d'un résidu fortement décarbonaté. Cette redistribution des carbonates s'accompagne d'une profonde modification de la granulométrie du rhizocylindre, qui de limono-argileux devient sableux (fig. 8-2 et fig. 1c, photo 2).

\section{b) Action des lombriciens}

Rappelons que les lombriciens creusent leurs galeries par ingestion de matériau, qui est rejeté une fois la matière organique digérée. Ce transfert à travers le tube digestif du ver a pour conséquences, d'une part, un pétrissage, d'autre part, un transport du matériau. Sur lames minces et surfaces polies de sol imprégné, l'activité des lombriciens se manifeste dans le sol étudié par la présence de turricules de rejet, plus ou moins cylindriques, souvent d'aspect très différent du matériau en place (fig. 1c, photo 1 ).

Dans un deuxième temps, micro-structures ct matériaux marneux sont brassés et homogénéisés par les lombriciens : la structure sédimentaire des marnes disparaît tandis que les cellules calcifiées sont dispersées dans une matrice sablolimoneuse (fig. 8-3). Ainsi, l'action de ces vers, sensible dès $80 \mathrm{~cm}$ de profondeur, a désorganisé la quasi-totalité du volume des marnes entre $30 \mathrm{~cm}$ de profondeur et la surface du sol (fig. 8-4).

En définitive, les sédiments marneux originels s'altèrent sous l'effet conjugué de 2 mécanismes indépendants :

- une altération physico-chimique microstructuralc d'origine racinaire ayant pour effet une décarbonatation relative de la matrice et la genèse de sables calcaires cytomorphes ;

- une altération mécanique macro-structurale d'origine animale, ayant pour effet la désorganisation de la structure sédimentaire héritée et l'incorporation des cellules calcifiées à la matrice.

\section{CONCLUSION}

- L'accumulation de particules solides par ou dans les racines est un phénomène connu mais dont les implications pédologiques n'ont que rarement été prises en compte. L'action instantanée d'une racine est faible et celle d'un système racinaire modeste, mais leur action cumulée au cours du temps peut avoir des effets considérables. Le sol étudié constituc un bel exemple à ce sujet : 25 p. 100 au moins de la phase minérale a été transformée par les racines.

Un phénomène similaire a été observé en conditions expérimentales. CHEN et al. (1980) montrent qu'en milieu hydromorphe réducteur, les racines de riz induisent la précipitation d'hydroxydes ferriques sur leurs parois, voire à l'intéricur des cellules. Cette oxydation entraîne la genèse de particules d'hydroxydes ayant la forme et la taille des cellules de la racine de riz. Il se pourrait donc que de tels phénomènes soient plus fréquents et généraux qu'il n'y paraît.

Quoi qu'il en soit, la participation des êtres vivants du sol à la transformation du milieu au sein duquel ils vivent est une réalité en dehors de laquelle la compréhension du fonctionnement du sol peut n'être qu'approchéc, voire erronée.

- Le processus de calcification des structures végétales présente aussi un intérêt agronomique important. Lors d'un précédent travail (JAILLARD, 1983), nous avons signalé l'existence de structures rhizomorphes en milieu chlorosant, dans des sols bruns calcaires à horizon d'accumulation calcaire développés sur limons quaternaires. Or, ces mêmes structures étaient absentes à une dizaine de mètres de distance en milieu sain, le sol se caractérisant par une porosité plus élevée, le rendant plus perméable et mieux aéré. De même, à Saint-Gély-du-Fesc, les structures rhizomorphes, très abondantes dans les sols d'altération de marnes, sont en revanche absentes dans les sols développés sur les bancs calcaires marneux fissurés.

En conséquence, il semblerait que le processus de calcification des racines soit dépendant des conditions physicochimiques et du fonctionnement hydrodynamique régnant dans ces milieux carbonatés massifs, et peut être en relation avec la maladie physiologique propre aux milieux carbonatés qu'est la chlorose ferrique.

Reçu le 21 février 1983. Accepté le 6 septembre 1983.

\section{REMERCIEMENTS}

J'exprimerai ici ma reconnaissance à mon ami et responsable de recherche Gabriel CALLOT, Maître de Recherche à I'I.N.R.A. : c'est lui qui a effectivement tenté de me convaincre que les végétaux, tout comme les champignons, pouvaient contribuer de manière significative à la transformation de la phase minérale du sol. Cette conception du sol est à l'origine de ce travail.

Je tiens à remercier mon camarade Michel MEYER, inventeur du site, pour m'avoir fait part de son expérience de ce terrain et pour l'intérêt qu'il porte à ce prolongement inattendu de son travail.

Je remercierai également Gérard Souche pour son efficace collaboration technique.

\section{RÉFÉRENCES BIBLIOGRAPHIQUES}

Butel P., 1982. Formes et mécanismes de l'accumulation carbonatée dans les sols de la plaine poitevine. Thèse de $3^{\mathrm{e}}$ cycle, Univ. de Poitiers, déc. 1982, $122 \mathrm{p}$.

Callot G., Chamayou H., Maertens C., Salsac L., 1983. Les interactions sol-racine. Incidence sur la nutrition minérale, I.N.R.A., Paris, 360 p.

Chen C., Dixon J. B., Turner F. T., 1980. Iron coatings on rice roots. Morphology and models of development. Soil Sci. Soc. Am. J., 44 (5), 1113-1119.

Chino M., 1981. Species differences in calcium and potassium distributions within plant roots. Soil Sci. Plant Nutr., 27 (4), 487503.
Ghorbal M. H., 1979. Absorption du calcium, localisation et rôle dans la perméabilité membranaire. Relation avec le caractère calcicole et calcifuge. Thèse de Doct. d'Etat, Montpellier, $402 \mathrm{p}$.

Jaillard B., 1983. Relation entre dynamique de l'eau et organisation morphologique d'un sol calcaire. Sci. Sol, 1, 31-52.

Meyer M., 1981. Eléments d'analyse géo-pédologique du domaine expérimental de Coulondres, commune de Saint-Gély-du-Fesc (Hérault). D.E.A., USTL-Montpellier, 2 octobre 1981, 31 p.

Wieder M., Yaalon D. H., 1982. Micromorphological fabrics and developmental stages of carbonate nodular forms related to soil characteristics. Geoderma, 28 (3/4), 203-220. 